Check for updates

Cite this: RSC Adv., 2017, 7, 49463

\title{
Changes in physical and chemical properties of niosome membrane induced by cholesterol: a promising approach for niosome bilayer intervention
}

\author{
Mohammad Hadi Nematollahi, (D) ab Abbas Pardakhty, ${ }^{a}$ Masoud Torkzadeh-Mahanai, ${ }^{\mathrm{C}}$ \\ Mehrnaz Mehrabani ${ }^{d}$ and Gholamreza Asadikaram ${ }^{* b e}$
}

Recently, the self-assembly property of nonionic surfactants has been utilized to create vesicles as alternatives to liposomes. The aims of the present study were to investigate the impact of cholesterol on niosome bilayer properties and to evaluate niosome solubilization by Triton X-100 (TR) in the presence and absence of cholesterol. Niosomes were prepared using Span 80 and Tween 80 with or without cholesterol, and several structural studies were done. We compared both types of niosomes in their rigidity, permeability, surface tension, thermal properties, toxicity profile, and solubilization by the addition of several aliquots of TR. Efforts to achieve solubilizing parameters are also discussed. Our results indicate that cholesterol in niosomes increases membrane stability, decreases the fluidity of the membrane and also alters membrane permeability. Additionally, the solubilization process is illustrated by a three-stage model, in which the vesicle-to-micelle transition for niosomes is induced by the stepwise addition of TR. We show that cholesterol-containing niosomes need a higher amount of TR concentration for solubilization. To our best knowledge, this is the first report on niosome solubilization with TR. The results revealed the similarity of niosomal to liposomal systems, and we hope this encourages researchers to design more solubilization and protein reconstitution studies with niosomes.

\author{
Received 16th July 2017 \\ Accepted 9th October 2017 \\ DOI: $10.1039 / c 7 r a 07834 j$ \\ rsc.li/rsc-advances
}

\section{Introduction}

Nowadays, vesicular systems have become very important tools in biochemical and pharmaceutical applications. These vesicular bilayers are lamellar structures containing amphiphilic molecules that entrap an aqueous compartment ${ }^{1}$ and might be employed in the delivery of hydrophilic (encapsulated in the interior hydrophilic compartment) and hydrophobic (encapsulated in the lipid layer) drugs. ${ }^{1}$ Virosomal vaccine and protein insertion into the vesicle bilayer are other vesicle applications that can occur in a reconstitution procedure. ${ }^{2}$ Thus, it can be considered that one of the potent approaches to characterize the functionality of integral proteins is their reconstitution into the phospholipid vesicles, and it is important to note that the

${ }^{a}$ Pharmaceutics Research Center, Institute of Neuropharmacology, Kerman University of Medical Science, Kerman, Iran

${ }^{b}$ Department of Biochemistry, School of Medicine, Kerman University of Medical Sciences, Kerman, Iran. E-mail: gh_asadi@kmu.ac.ir

${ }^{c}$ Biotechnology Department, Institute of Science and High Technology and Environmental Sciences, Graduate University of Advanced Technology, Kerman, Iran ${ }^{d}$ Physiology Research Center, Institute of Basic and Clinical Physiology Sciences, Kerman University of Medical Sciences, Kerman, Iran

${ }^{e}$ Neuroscience Research Center, Institute of Neuropharmacology, Kerman University of Medical Sciences, Kerman, Iran biological activity of the integral protein must remain high enough. ${ }^{3,4}$

The membrane proteins can insert into liposomes through various ways. In this regard, four key approaches can be found according to previous studies, including mechanical means, freeze-thawing, organic solvents, and detergent-mediated insertion. However, the first three methods have technical limitations, and the most common approach is introducing detergent to the liposome bilayer. ${ }^{3,5}$ The biological membrane solubilization caused by detergents is an important process in the biochemical and biophysical studies on the insertion of purified protein in a bilayer membrane, ${ }^{4,6}$ and a lot of studies have suggested techniques to remove the detergents after protein reconstitution, involving dialysis, column chromatography or incubation with detergent-adsorbing beads. ${ }^{7}$

Solubilization of phospholipid vesicles by detergents has been considered to occur in three general stages. At low detergent concentrations, the detergent partitions into the vesicle structure. When the vesicle bilayer becomes saturated with detergent, the system undergoes a structural transition from vesicular structure to mixed micelles. As detergent concentrations are further increased, vesicles become fully solubilized by the detergent. ${ }^{8}$ It is well known that some parameters, such as temperature, pressure, lipid concentration and lipid 
composition, influence the solubilization process. ${ }^{\text {9,10 }}$ Among these factors, lipid composition is one of the most important issues. In biological membranes, cholesterol, as the main sterol, has a significant role in the physical features of the lipid bilayers. ${ }^{11}$ Furthermore, it should be mentioned that the physical status of the bilayers can affect vesicular solubilization. ${ }^{12}$ The orientations of the $3 \beta$-hydroxyl and hydrophobic groups of cholesterol are, respectively, toward the polar and nonpolar head group regions of the phospholipid bilayer, affecting the formation of intermolecular hydrogen bonds. ${ }^{13}$ Previous reports indicate that cholesterol can alter membrane properties, and the amount of detergent needed for membrane solubilization can be changed by cholesterol, as well. ${ }^{\mathbf{1 4 - 1 9}}$

Due to the short stability and cost-effective production of liposomes, finding an alternative vesicular system with similar properties to liposomes is a greatly interesting field. ${ }^{20,21}$ Niosomes or nonionic surfactant-based vesicles form from the selfassembly of amphiphiles in the aqueous solution, which leads to the formation of closed bilayer structures. These structures can encapsulate aqueous solutes and serve as drug carriers, as analogous to liposomes. ${ }^{21}$ The physical characteristics of these vesicles are the same as liposomes; in addition, their preparation method is identical and their unilamellar or multilamellar structures are similar to liposomes. Although the first application of niosomes was in the cosmetic industry, ${ }^{22}$ the low cost, better stability, and easy storage of the nonionic surfactants led to the utilization of these compounds as an alternative to phospholipid-containing vesicles. ${ }^{23}$ Subsequently, a large number of niosome applications in drug delivery, gene delivery, and vaccine design have been assessed. ${ }^{23}$ Hence, characterization of the niosome bilayer is crucial for expanded application of niosomes. To our best knowledge, there has been no investigation on niosome solubilization with TR and detergent removal to date.

Therefore, to find a good alternative for liposome applications, we planned to study some biophysical properties of the unsaturated niosome bilayer with or without cholesterol. Although there are several reports on saturated and unsaturated liposome solubilization, ${ }^{24,25}$ we lack detailed knowledge on the solubilization of niosome bilayers. Therefore, in the next section, we aimed to study the solubilization of niosomes (with or without cholesterol) by the addition of increasing amounts of TR, using a similar process called a three-stage model previously reported.,26 Finally, detergent removal from the mixed micelle vesicle and vesicle re-formation were investigated.

\section{Materials and methods}

\subsection{Materials}

The cell line was obtained from Pasteur Institute of Iran (Tehran, Iran). Sorbitan monooleate (Span 80), polyoxyethylene sorbitan monooleate (Tween 80), cholesterol, Triton X-100 and thiazolyl blue tetrazolium blue (MTT) powder were purchased from Sigma-Aldrich (Steinheim, Germany). Nile red (NR) was provided by Santa Cruz Biotechnology (Santa Cruz, CA, USA), and calcein, cobalt chloride, fetal bovine serum (FBS), and Dulbecco's Modified Eagle's Medium (DMEM) culture media were obtained from Thermo Scientific (Carlsbad, CA, USA). All other materials were taken from available sources with the highest purity.

\subsection{Niosome preparation}

Nonionic surfactant mixtures consisting of Span 80 and Tween 80 with or without cholesterol were used to prepare niosomes by a thin film hydration (TFH) method, at specified ratios as summarized in Table 1 . In brief, surfactants and cholesterol were dissolved in chloroform $\left(50 \mathrm{mg} \mathrm{ml}^{-1}\right)$. The specific lipid compositions were then transferred to a $5 \mathrm{ml}$ round-bottom flask. A rotary evaporator apparatus was used under negative pressure to evaporate the solvent and subsequently form the thin lipid film. The formed film was hydrated with $1 \mathrm{ml}$ of 3-morpholinopropane-1-sulfonic acid (MOPS) (10 mM, pH 7.4). ${ }^{27,28}$

\subsection{Biophysical membrane experiments}

2.3.1. Membrane rigidity. Although Nile red (NR) dye has low fluorescence intensity in a polar environment, it emits a strong signal when incorporated into hydrophobic membranes. The value of the fluorescence intensity indicates the degree of membrane rigidity. This property was used to measure the rigidity of the ST80 and ST80CH niosome bilayers. Likewise, NR maximum emission shift was considered as another marker for membrane rigidity. Fluorescence intensity was measured after the addition of $0.4 \mu \mathrm{g} \mathrm{ml}{ }^{-1} \mathrm{NR}$. The measurements were recorded at $25{ }^{\circ} \mathrm{C}$ using a Cary Eclipse fluorescence spectrophotometer (Varian, Palo Alto, CA). The excitation wavelength was $550 \mathrm{~nm}$, and emission was monitored between 560-700 nm. NR emission in MOPS buffer was considered as a background fluorescence signal. ${ }^{29,30}$

2.3.2. Surface tension measurements. Niosomes were prepared as described earlier, and surface tension of ST80 and ST80CH niosomes was measured by the Du Noüy ring method using a Krüss Tensiometer (Model K100, Germany) at $25^{\circ} \mathrm{C}$. Ten measurements were done for each formulation, and mean \pm SD is reported.

2.3.3. Differential scanning calorimetry (DSC). Thermal behaviors of unsaturated niosomes with or without cholesterol were monitored using the PerkinElmer Pyris 6 differential scanning calorimeter (PerkinElmer, USA). The heating run was performed from $-40{ }^{\circ} \mathrm{C}$ to $250{ }^{\circ} \mathrm{C}$ with a heating rate of $5{ }^{\circ} \mathrm{C} \min ^{-1}$. The temperature at the peak maximum was considered as the phase transition temperature. ${ }^{31}$

2.3.4. Niosome bilayer permeability. Membrane permeabilization of niosomes was evaluated using a leakage assay as described previously by Kendall et al. ${ }^{32}$ Briefly, the calceincobalt complex with low fluorescent signal was encapsulated

Table 1 Niosome formulations used in the study

\begin{tabular}{lll}
\hline Niosome formulation & Molar ratio & Abbreviation \\
\hline Span 80/Tween 80 & $50 / 50$ & ST80 \\
Span 80/Tween 80/cholesterol & $40 / 40 / 20$ & ST80CH
\end{tabular}


into niosomes. As calcein-cobalt leaks from the niosomes, the external EDTA binds cobalt, and free calcein fluorescence increases. Fluorescence was monitored using a Fluorolog 3 (Horiba-JobinYvon) fluorimeter under continuous stirring at $25{ }^{\circ} \mathrm{C}$. Measurements were taken for $6 \mathrm{~h}$ at specific intervals.

\subsection{Bioactivity membrane experiments}

2.4.1. Niosome cytotoxicity. The HEK 293T cell line was cultured in DMEM medium with $10 \%$ FBS and 1\% antibiotic (Pen-Strep). Cells were seeded in 96-well plates and incubated in a $\mathrm{CO}_{2}$ incubator at $37^{\circ} \mathrm{C}$. Different unsaturated niosomes (with or without cholesterol) at various concentrations were prepared and evaluated for their toxicity to cells by performing the MTT assay. The MTT assay was conducted essentially as in our previous study. ${ }^{33}$

2.4.2. Hemolysis. Two milliliters of sheep red blood cells (prepared from Pasteur Institute, Tehran, Iran) was washed several times with physiological ionic strength $\mathrm{NaCl}$ solution, counted, and then resuspended in the same buffer at specific cell counts and at $\mathrm{pH}$ 7.4. The mixtures were incubated at $37^{\circ} \mathrm{C}$ for $1 \mathrm{~h}$ and centrifuged at $2000 \mathrm{~g}$ for $10 \mathrm{~min}$ at $4{ }^{\circ} \mathrm{C}$, and the supernatant absorbance was measured at $541 \mathrm{~nm} .^{34}$

\subsection{Solubilization experiments}

2.5.1. Niosome titration. Preformed and presized niosome suspensions were mixed with increasing amounts of the TR solutions. Final niosome concentrations were 1, 2, 5 and $10 \mathrm{mM}$. After each aliquot reached a steady state, the vesicles and detergent were left at room temperature (RT) for $1 \mathrm{~h}$ to equilibrate, and the solubilization was monitored by the changes in turbidity. ${ }^{35}$ Differences were measured as absorbance at $540 \mathrm{~nm}$ in a Cary $500 \mathrm{UV}$-VIS-NIR spectrophotometer (Varian, Australia).

2.5.2. Solubilization parameters. Routinely, solubilization was characterized in six terms, including $R_{\mathrm{e}}^{\mathrm{sat}}, R_{\mathrm{e}}^{\mathrm{sol}}, D_{\mathrm{w}}^{\mathrm{sat}}, D_{\mathrm{w}}^{\mathrm{sol}}, D_{\mathrm{t}}^{\mathrm{sat}}$ and $D_{\mathrm{t}}^{\mathrm{sol}} \cdot{ }^{26,36,37}$ The detergent-to-lipid ratio at the greatest saturation $\left(R_{\mathrm{e}}^{\mathrm{sat}}\right)$ and total solubilization points $\left(R_{\mathrm{e}}^{\mathrm{sol}}\right)$ were determined by the slopes of two phase boundary straight lines. These phase boundaries can be detected using light scattering measurements. $D_{\mathrm{w}}^{\mathrm{sat}}$ and $D_{\mathrm{w}}^{\mathrm{sol}}$ are the detergent concentrations at the onset of saturation and solubilization, correspondingly, and they are actually lower than the critical micelle concentration (CMC) of the pure detergents. The free detergent concentrations in water at the onset and completion of solubilization $\left(D_{\mathrm{w}}^{\mathrm{sat}}\right.$ and $D_{\mathrm{w}}^{\mathrm{sol}}$, respectively) are obtained from the intersection of the phase boundaries with the $Y$-axis. ${ }^{38} D_{\mathrm{t}}^{\text {sat }}$ and $D_{\mathrm{t}}^{\text {sol }}$ are the aqueous detergent concentrations of the surfactant monomers corresponding to $R_{\mathrm{e}}^{\text {sat }}$ and $R_{\mathrm{e}}^{\mathrm{sol}}$, respectively.

2.5.3. Removal of Triton X-100 with Bio-Beads SM-2. BioBeads SM-2 preparation was done according to the previous report. $^{2}$ For detergent removal, four aliquots of wet Bio-Beads SM-2 were added at certain intervals. Bio-Beads SM-2 was removed from the solution by filtration using disposable polyprep columns, and the reconstituted niosomes were collected by centrifugation $(30 \mathrm{~min}$ at $23000 \mathrm{~g})$. $^{2,5}$

\subsection{Vesicle-to-micelle transition}

2.6.1. Assessment of niosome solubilization by dynamic light scattering. Niosome solubilization by TR was monitored by dynamic light scattering (DLS) as described by Xu and London. ${ }^{17}$ SPL method was used to analyze the size changes during TR addition. Each sample was measured in triplicate using the VASCO $^{\mathrm{TM}}$ size particle analyzer (Cordouan, France). The size measurements are reported as mean $\pm \mathrm{SD}(n=3)$.

2.6.2. Assessment of niosome solubilization by fluorescence intensity. Based on the previous report, NR is a useful probe to determine the vesicle-to-micelle transition. ${ }^{39}$ According to our preliminary experiments, a methanolic stock solution of $\mathrm{NR}(2.5 \mathrm{mM})$ was used and was added to a $0.25 \mathrm{mM}$ niosome solution at a ratio of $1: 2000$. NR fluorescence emission was measured with each TR addition on a Cary Eclipse fluorescence spectrophotometer (Varian, Palo Alto, CA) at $25{ }^{\circ} \mathrm{C}$, using an excitation wavelength of $550 \mathrm{~nm} .^{24,39,40}$

\subsection{Micelle-to-vesicle transition}

2.7.1. Scanning electron microscopy (SEM) analysis. ST80 and ST80CH niosomes were solubilized by TR and then treated with Bio-Beads to remove TR. Another niosome solution without solubilization was sent to the SEM lab and considered as a control group. The morphology of niosomes was observed by SEM (KYKY SEM China; EM-3200).

2.7.2. TR tracing by gas chromatography-mass spectrometry (GC-MS). To confirm complete TR removal, GC-MS (Agilent Technologies, Carian Saturn 2000, USA) was carried out. The detergent was detected according to the method previously described by Poss et al. ${ }^{41}$ The GC-MS analysis was performed using a Varian Saturn 2000/HP5973 GC-MS system (Agilent Technologies, Santa Clara, CA, USA).

\subsection{Data analysis}

Statistical analysis was done using Student's $T$ test or one-way analysis of variance (ANOVA), followed by Tukey post hoc test using SPSS 16 software. $P$ values $<0.05$ were considered significant.

\section{Results and discussion}

\subsection{Niosome formation}

The formation of vesicular structures related to ST80 and ST80CH niosomes was confirmed by light microscope to give qualitative data (not shown) concerning the size and shape of the niosomes. The details of niosome formulations are summarized in Table 1 . Niosomes were prepared successfully using both formulations.

\subsection{Membrane rigidity, surface tension, DSC and bilayer permeability results}

It is well known that the rigidity of the membrane influences the fluorescence emission of many lipid dyes. Principally, in a polar environment, the fluorescence intensity of NR is low, but incorporation into hydrophobic cell membranes leads to strong 
emission, guiding us toward measurement of the membrane integrity in niosome membrane. ${ }^{30}$ To test the rigidity difference between ST80 and ST80CH vesicles, we measured the fluorescence intensity of NR. According to our results and based on Kucherak et al. 's study, ${ }^{30}$ ST80 and ST80CH niosomes were in Ld phase, but there were differences in their fluidity maximum emission and their fluorescence intensity. Binding of the NR dye to ST80 and ST80CH niosomes caused an about 160 -fold and 125-fold increase in NR fluorescence intensity compared to $\mathrm{NR}$ in the buffer, respectively. The fluorescence intensity in ST80 compared to ST80CH was lower, showing a difference in their membrane rigidity (Fig. 1a). The maximum NR emission is presented in Fig. 1b. Our results revealed that the emission shift of ST80CH is stronger compared to ST80, and this dye could detect the rigidity differences between niosomes. ST80CH niosomes had more rigid membrane compared to ST80 niosomes, showing that cholesterol insertion in the niosome membrane decreases the membrane fluidity, compared to previously reported niosome compositions and liposomes. ${ }^{\mathbf{1 6 , 2 1 , 4 2}}$

Span 80 and Tween 80 have very low transition temperatures due to the unsaturated double bonds in their oleate side chains. Thus, a high leakage is predictable for cholesterol-free niosomes. We evaluated leakage by calcein-cobalt assay. According to Fig. 1c, cholesterol-free niosomes had significant leakage compared to cholesterol-containing niosomes $(P<0.05)$. Incorporation of cholesterol caused a more ordered membrane and decreased the leakage. The leakage of ST80CH vesicles at $25{ }^{\circ} \mathrm{C}$ was four-fold lower than that of ST80 niosomes, revealing that incorporation of cholesterol into the niosomes leads to the lower amount of vesicle leakage. Decreases in leakage occur through the formation of hydrogen bonds between the hydroxyl groups of cholesterol and the oxygen at the ester groups, or with the other functional oxygen groups of Span 80, thus enhancing the stability of the bilayer. ${ }^{16}$ It has been previously reported that
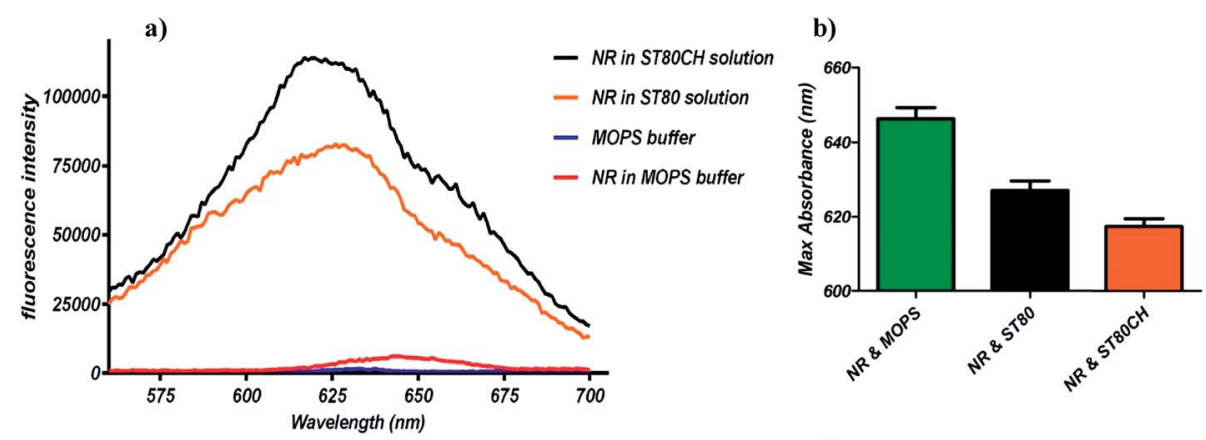

c)

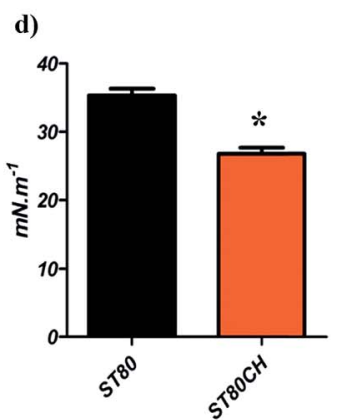

e)

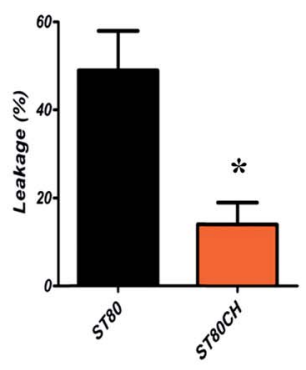

f)
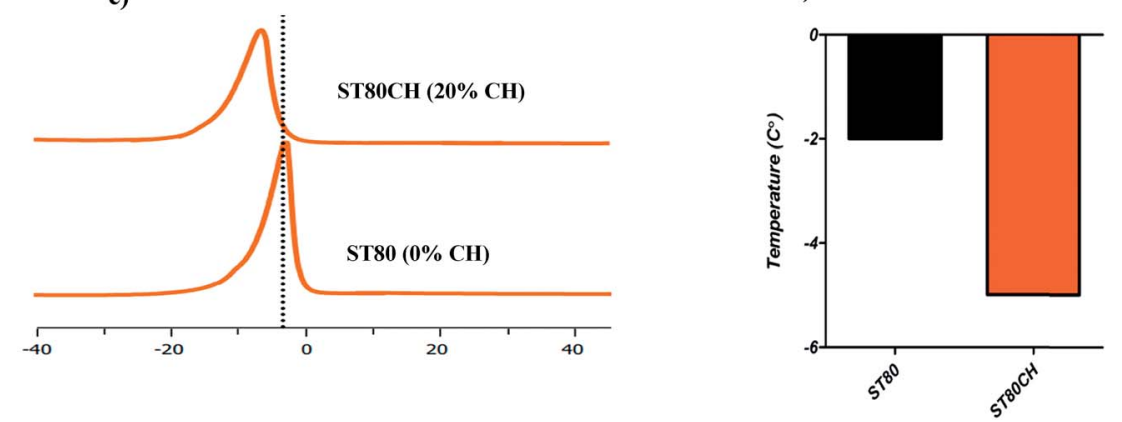

Fig. 1 Niosome membrane characterization. (a) Membrane rigidity was measured by NR probe. ST80 has lower intensity compared to ST80CH. (b) Maximum emission of NR was considered as a rigidity index. (c) ST80 and ST80CH niosome leakage after $4 \mathrm{~h}$ monitored by calcein-cobalt method. Cholesterol insertion in ST80 CH had significant effect on vesicle leakage. Data represent the mean values of 3 experiments. $* P<0.001$. (d) Cholesterol incorporation (20\%) decreased the surface tension significantly. Data represent the mean values of 10 measurements. $* P<0.05$. (e) DSC thermograms of ST80 and ST80CH niosomes showing the effect of cholesterol on the $L_{\beta} / L_{\alpha}$ phase transition. (f) The cholesterol concentration indicated in the figure. The shift of $T_{m}$ after cholesterol insertion is depicted. 
including cholesterol in liposomes decreased the leakage of encapsulated content. ${ }^{16,43}$

To find the maximal stability and niosome functionality, surface tension of the niosome should be estimated. The surface tension of niosomes is related to the surfactant and cholesterol content, and our results revealed that cholesterol insertion in the niosome bilayer decreases surface tension (Fig. 1d). ${ }^{44}$ The surface tension difference between ST80 and ST80CH was found significant $(P<0.05)$. As Azarbayjani et al. and also Vargha-Butler et al. have reported, a decrease in the surface tension value with increasing cholesterol content indicates that the surface of the bilayer becomes less hydrophilic, which leads to increased membrane integrity. ${ }^{4,45}$

A useful technique to study the impact of cholesterol on the phase transition of vesicles is DSC. ${ }^{\mathbf{1 9 , 4 2 , 4 6}}$ Fig. 1e depicts DSC results that are in good agreement with those of previous reports on liposome ${ }^{47}$ and niosome ${ }^{42}$ as a function of cholesterol content. In our results, a broadening and decreasing in enthalpy was observed in ST80CH niosome bilayers. In addition to the decrease in enthalpy, the ST80CH vesicles exhibited a phase transition temperature shift $\left(\sim 3{ }^{\circ} \mathrm{C}\right)$ to lower temperatures as cholesterol was incorporated in the bilayer (Fig. 1f). The interaction of cholesterol with the single-chain nonionic surfactants and its impact on the gel-to-liquid crystalline transition of the niosomes was studied with the help of DSC. An order-disorder transformation (phase transition) occurs in the alkyl chains of the nonionic surfactant with increasing phase transition temperature $\left(T_{\mathrm{m}}\right) \cdot{ }^{48}$ During this process, some variations can be seen in the bilayer properties such as permeability and fluidity. ${ }^{49}$ The alkyl chains that are fluid originally are highly permeable to small molecules above their gel-to-liquid crystalline transition temperature. ${ }^{42}$ The temperature, lipid composition, and presence of other components such as cholesterol can affect the gel-to-liquid crystalline phase transition. A previous study has shown that the inclusion of cholesterol $>30$ mole $\%$ abolished the gel-to-liquid transition of

a)

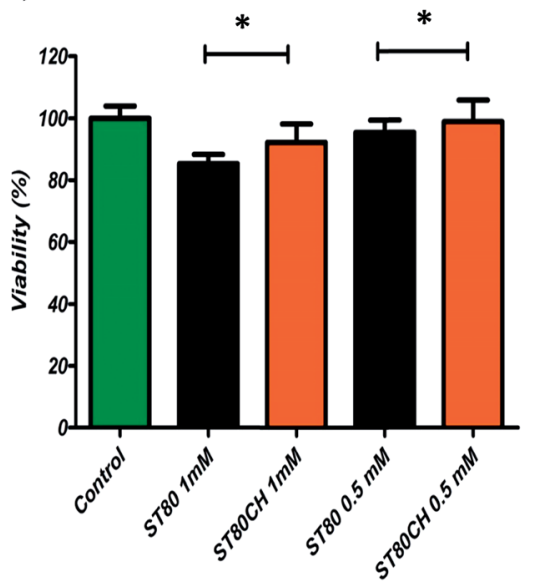

niosomes. ${ }^{50}$ Our results are in agreement with the results of cholesterol insertion in liposomes and niosomes.

\subsection{Niosome cytotoxicity and hemolysis}

Fig. 2b shows the relative hemolysis (\%) induced by the ST80 and ST80CH niosomes. As can be seen, in all cases, the hemolysis induced by vesicles was at low levels. TR-induced hemolysis was considered as $100 \%$ hemolysis, and MOPS as $0 \%$ hemolysis. As shown in Fig. 2a, cytotoxicity assays were performed using HEK293 cell line. The cytotoxic effects of niosomes increased with the higher concentration of niosomes. Another finding was that cholesterol addition reduced hemolysis and cytotoxicity. This difference was found significant at $P$ $<0.05$. Unfortunately, there are not an adequate number of niosome toxicity studies in the published literature, especially on the impact of cholesterol on cell viability. However, toxicity of ether and ester-type surfactants toxicity has been surveyed previously. ${ }^{51}$ Hofland and colleagues have revealed through cellular toxicity studies that niosomes composed of ester-type surfactants (Spans and Tweens) are less toxic than ether-type ones (Brijs). This might be related to the enzymatic degradation of ester bonds. ${ }^{51}$ In another study, Span 80 was found to have the least hemolytic activity among other sorbitan esters. ${ }^{52}$ Hofland et al. found that the cholesterol content of the bilayers do not affect cell proliferation. ${ }^{51}$ However, in the present study, we showed that insertion of cholesterol in vesicles can significantly decrease the cytotoxic and hemolytic effects of the vesicles.

\subsection{Niosome solubilization and vesicle reconstitution}

The solubilizing parameters are given in Table 2, and phase boundaries are depicted in Fig. 3. Results show that cholesterol increases the coexistence region that is related to increased $R_{\mathrm{e}}^{\mathrm{sat}}$ and, especially, $R_{\mathrm{e}}^{\mathrm{sol}}$ values. The three stages of detergent niosome solubilization are depicted as changes in turbidity measurements on LUVs (large unilamellar vesicles) with b)

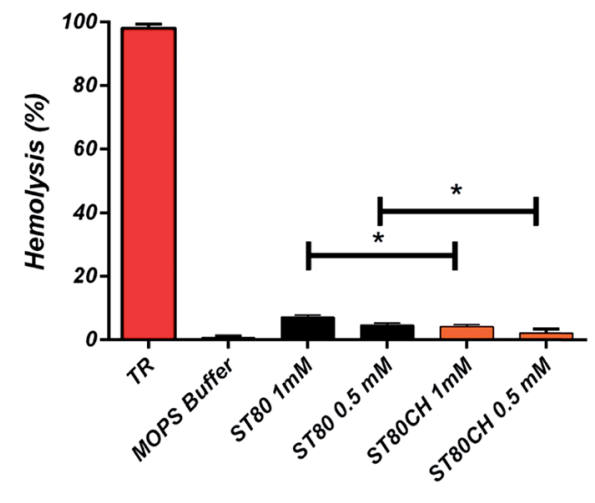

Fig. 2 Biological properties of niosomes. (a) Cellular cytotoxicity profile of niosomes with and without cholesterol are depicted. Data represent the mean values of 3 measurements. $* P<0.05$. (b) Hemolysis profile of niosomes with and without cholesterol. Data represent the mean values of 3 measurements. $* P<0.05$. Triton $X-100$ and MOPS buffer considered $100 \%$ and zero percent hemolysis, respectively. 
Table 2 The effect of cholesterol on the solubilization of ST80 and ST80CH bilayers by Triton $\mathrm{X}-100$. See text for the meaning of the different parameters

\begin{tabular}{lcclllr}
\hline $\begin{array}{l}\text { Niosome } \\
\text { formulation }\end{array}$ & $R_{\mathrm{e}}^{\text {sat }}$ & $R_{\mathrm{e}}^{\text {sol }}$ & $\begin{array}{l}D_{\mathrm{w}}^{\text {sat }} \\
(\mathrm{mM})\end{array}$ & $\begin{array}{l}D_{\mathrm{w}}^{\text {sol }} \\
(\mathrm{mM})\end{array}$ & $\begin{array}{l}D_{\mathrm{t}}^{\text {sat }} \\
(\mathrm{mM})\end{array}$ & $\begin{array}{l}D_{\mathrm{t}}^{\text {sol }} \\
(\mathrm{mM})\end{array}$ \\
\hline ST80 & 0.29 & 0.51 & 0.08 & 0.15 & 3.2 & 5.7 \\
ST80CH & 0.57 & 1.31 & 0.16 & 0.23 & 6.6 & 11.9 \\
\hline
\end{tabular}
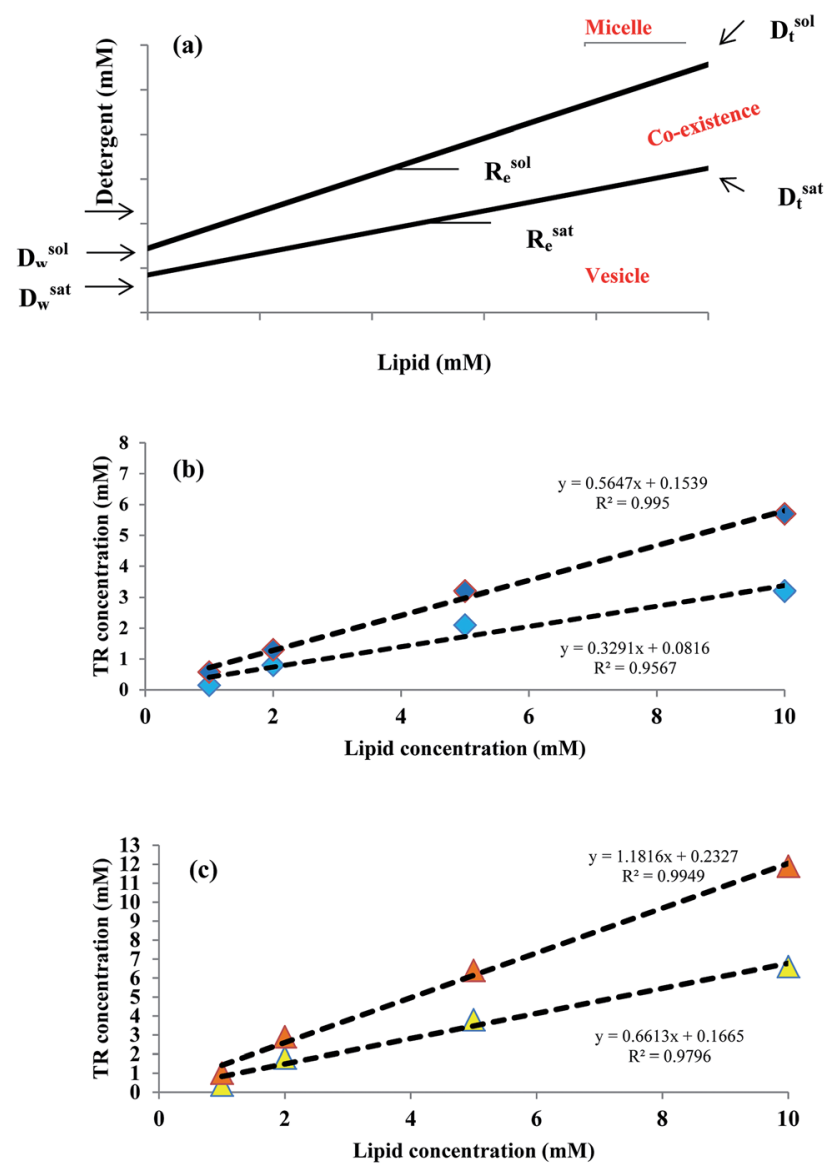

Fig. 3 Phase diagram for aqueous mixtures of niosomes and TR. (a) Schematic phase diagram of a mixed lipid-detergent system. Accordingly, the two lines represent the boundaries of the range of lipid and detergent concentrations where mixed vesicles coexist with mixed micelles. (b) Phase boundaries related to ST80 niosome solubilization by TR. (c) Phase boundaries related to ST80 CH niosome solubilization by TR.

increasing TR concentrations. The representative solubilization process for a single niosome and turbidity changes with TR addition during solubilization are illustrated in Fig. $4 \mathrm{a}$ and b, respectively. In stage I, a low concentration of detergent results in less incorporation in the membrane bilayer, without causing any substantial alteration in the vesicle structure. Higher detergent concentration and insertion of the detergent into the bilayer lead to initial swelling of the niosomes. Thus, in this solubilization step, the turbidity of ST80 and ST80CH vesicles increased due to the incorporation of TR into the niosome a)

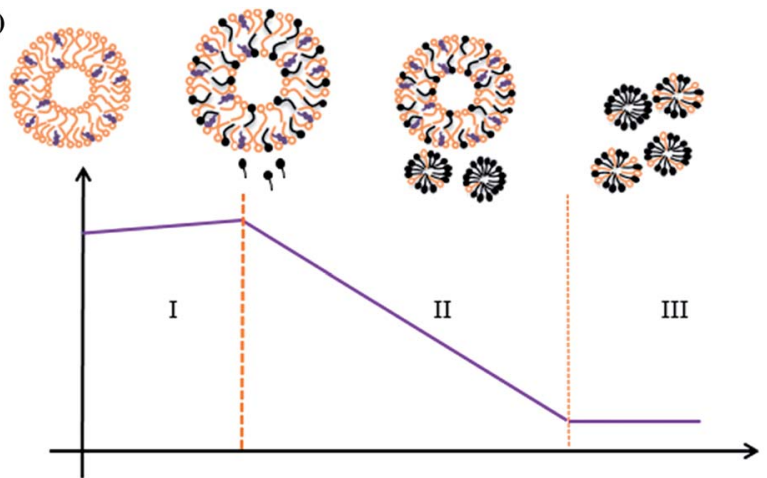

b)

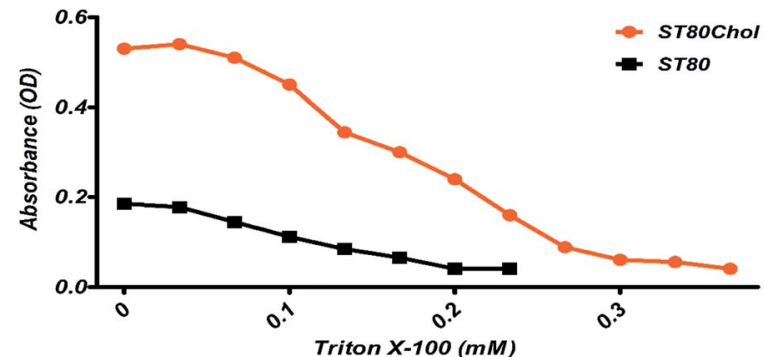

Fig. 4 (a) Schematic of niosome titration with TR and different structures that may occur during the solubilization process, i.e. the transition from niosome vesicles to mixed micelles. (b) Destabilization of phospholipid vesicles with Triton X-100, monitored by turbidity. As a measure of turbidity, optical density at $540 \mathrm{~nm}$ is plotted versus detergent concentration. Niosome concentration was $1 \mathrm{mM}$ for both ST80 and ST80CH.

bilayer membrane. Increased detergent concentration resulted in bilayer saturation and, consequently, mixed micelle formations (stage II in Fig. 4a). The maximum absorbance stays constant until $R_{\mathrm{e}}^{\mathrm{sat}}$ is reached (at 0.29 and 0.57 for ST80 and ST80CH, respectively), and TR concentrations corresponding to $R_{\mathrm{e}}^{\text {sat }}\left(D_{\mathrm{t}}^{\text {sat }}\right)$ are $3.2 \mathrm{mM}$ and $6.6 \mathrm{mM}$ for ST80 and $\mathrm{ST} 80 \mathrm{CH}$, respectively. Further detergent addition caused more mixed micelle formation, indicated by the decrease in absorbance during the turbidity measurements. This decrease in the optical density reached a minimum at $R_{\mathrm{e}}^{\text {sol }}$, which marks the onset of solubilization (at 0.51 and 1.31 for ST80 and ST80CH, respectively, stage III in Fig. 4a). TR concentrations corresponding to $R_{\mathrm{e}}^{\mathrm{sol}}\left(D_{\mathrm{t}}^{\mathrm{sol}}\right)$ were $5.7 \mathrm{mM}$ and $11.9 \mathrm{mM}$ for ST80 and ST80CH, respectively. In ST80 niosomes $(5 \mathrm{mM})$, solubilization was completed at a total detergent concentration $\left(D_{\mathrm{t}}^{\text {sol }}\right)$ of $3.2 \mathrm{mM}$, a value much lower $(<2)$ compared to niosomes containing cholesterol. The solubilization data show that cholesterol significantly increased the required TR concentration in the aqueous medium for solubilization. Similar results were reported by Ahyayauch et al. on liposome models. ${ }^{4}$

\subsection{Vesicle-to-micelle transition}

For solubilization with TR, a substantial transition from vesicle to micelle was tracked by NR probe. In ST80 niosomes, the maximum emission wavelength $(\lambda)$ of NR changed from $627 \mathrm{~nm}$ for a vesicular niosome to $629 \mathrm{~nm}$ for the micellar form (Fig. 5a). 
a)
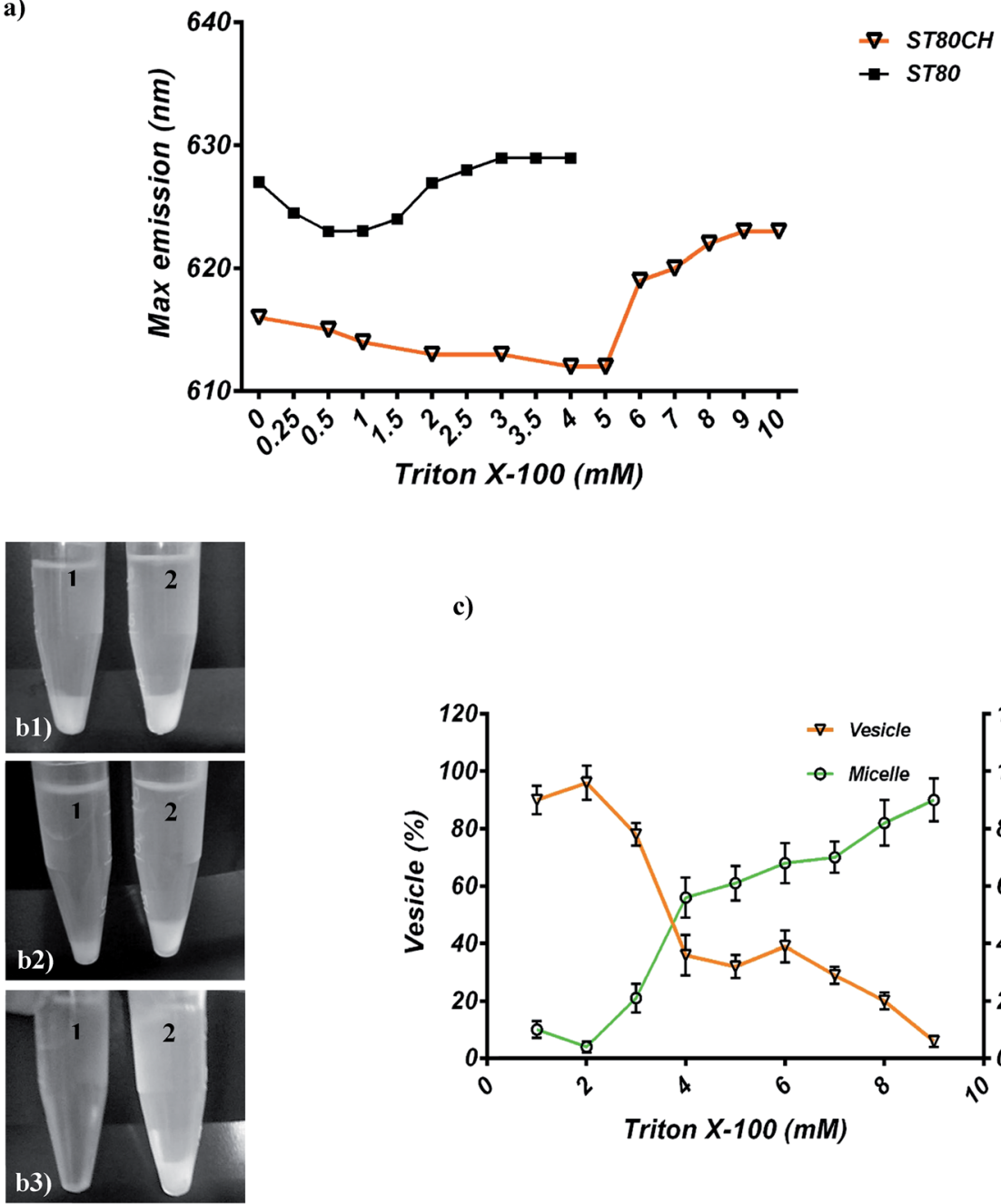

c)

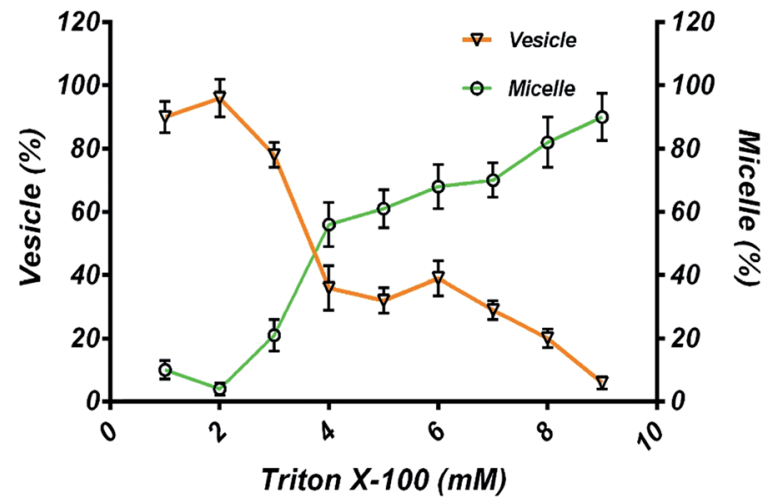

Fig. 5 (a) Vesicle-to-micelle transition monitored by Nile red fluorescence (max emission) on $0.25 \mathrm{Mm}$ ST80 and $0.25 \mathrm{Mm}$ ST80CH niosomes. (b) ST80CH niosomes were monitored to complete solubilization. (b1) ST80CH niosomes (2 mM) without TR addition. (b2) TR (8 mM) was added only to tube 1. Tube 2 is a control without TR. (b3) Complete solubilization in tube 1 by adding $12 \mathrm{mM}$ TR; tube 2 is the control. (c) Variation in the particle size (\%) of the vesicles and micelles in the solubilization process versus the detergent concentration (mM) monitored by DLS. The niosome concentration was $2 \mathrm{mM}$.

In ST80CH vesicles, the maximum emission shift was greater than that of ST80 and changed from $616 \mathrm{~nm}$ for a niosome bilayer to $620 \mathrm{~nm}$ for micellar form (Fig. 5b). It has been proven that in the micelles, some water will penetrate between the hydrophilic head groups of the surfactant molecules, causing a higher $\lambda_{\max }$ emission of NR. In contrast, in a bilayer, the surfactant molecules are better packed, and water is more tightly excluded from the interface, reflected by a lower NR $\lambda_{\text {max }}{ }^{39}$

Moreover, both ST80 and ST80CH niosomes were solubilized completely by TR (ST80CH results are shown in Fig. 5c). The changes in the niosome size were evaluated for each TR addition by DLS (Fig. 5d). Niosome vesicles enlarged to a certain value, and then the diameter of vesicles gradually decreased. Moreover, when the TR concentration exceeded about $3 \mathrm{mM}$ (point 3), a new peak in the size distribution (45 nm) was observed that is related to the formation of surfactant-detergent mixed micelles. Continuously increasing detergent concentrations led to a progressive increase of this new peak and a decrease in the initial peak. Accordingly, from points 3 to 8 , the size of small and large particles slightly rose and fell, respectively.

For each lipid composition, the phase boundary diagram included three regions. One area corresponds to all the surfactants in the bilayer form (vesicles). Another region is related to all the lipids in the form of surfactant-detergent mixed micelles. These two regions are separated by a third area named coexistence region, in which both the bilayers and mixed micelles are found. As mentioned in above, we displayed that cholesterol increases the coexistence region, and this finding confirms the effect of cholesterol on niosomal membrane solubilization. Some researchers have shown that cholesterol affects the amount of detergent required to solubilize unsaturated PC-based liposomes. For instance, Schnitzer et al. revealed 
a)

b)
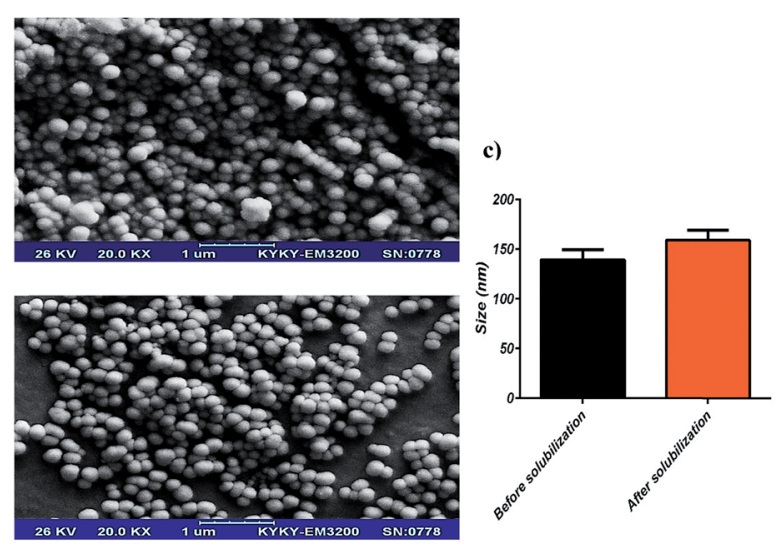

d1)

d2)

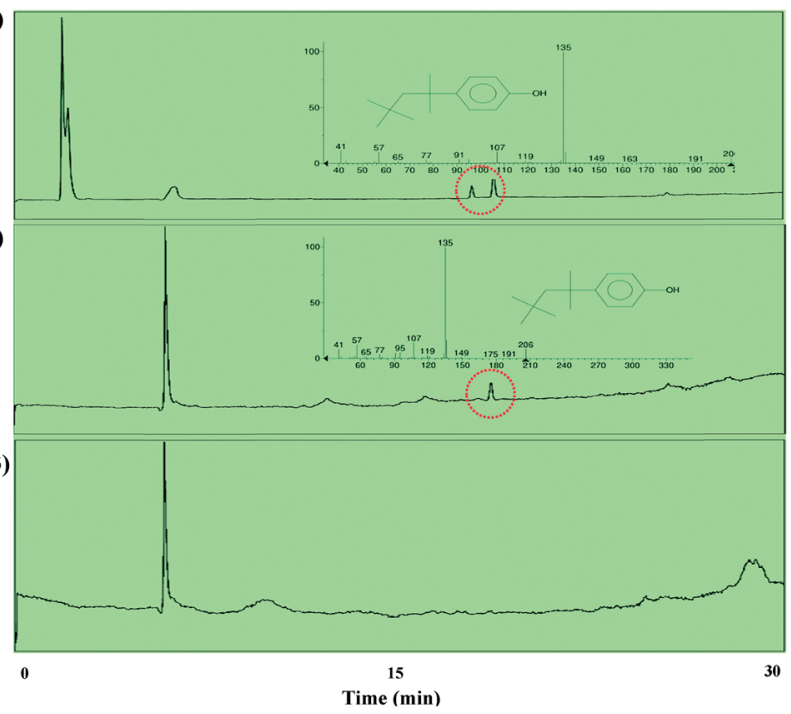

Fig. 6 (a) SEM image of ST80CH niosomes without TR addition. (b) SEM image of STCH80 after TR removal by Bio-Beads. Some surfactant loss can be observed. (c) Changes in size of niosomes after BioBeads treatment. Difference was not significant. (d) GC-MS chromatograms for TR detection in (d1) Triton X-100 as a control, (d2) ST80CH niosomes treated with TR, and (d3) ST80CH niosomes treated with TR and detergents removed by Bio-Beads. Time is 30 minutes. The related peak is indicated by a dotted circle.

that addition of cholesterol to unsaturated phospholipid containing vesicles increases the resistance of the bilayer to TR solubilization. ${ }^{14}$

\subsection{Micelle-to-vesicle transition}

Size homogeneity and unilamellarity of reconstituted vesicles are two well-known properties of re-formed vesicles that are related to the nature of detergent. It has been reported that re-formed vesicles (liposomes) by Bio-Beads are 150-200 $\mathrm{nm}$ in size. Our re-formed niosome sizes were in good agreement with previous reports $^{53,54}$ and had size homogeneity. Another set of data included in our study was the size changes in reconstituted niosomes (changes were not statistically significant). After detergent removal, niosomes had a size growth similar to that reported previously in liposome system (Fig. 6a-c). ${ }^{55}$ Although in other detergent removal methods (dialysis, gel chromatography, and dilution), size growth is related to residual detergent in the membrane, it is commonly believed that in Bio-Beads - mediated detergent removal, the growth is due to a fusion of the vesicles containing a large amount of detergent, as has previously been reported. ${ }^{56}$ Although there are various methods for detergent removal, including dialysis, gel chromatography, dilution and hydrophobic adsorption onto polystyrene beads (Bio-Beads SM-2), adsorption on Bio-Beads SM2 has been demonstrated to be the most efficient method. ${ }^{57}$ The first three methods are mostly limited to the use of detergents with a high CMC, such as n-octyl$\beta$-D-glucoside (OG); detergents with low CMC (like TR), which form larger micelles compared to OG, are not easily removed by dialysis, gel chromatography, or dilution but can be efficiently removed through adsorption on Bio-Beads SM-2 resins. ${ }^{57} \mathrm{~A}$ previous study has revealed that Bio-Beads can remove TR completely. ${ }^{57}$ In the GC-MS experiments, we showed that BioBeads SM-2 could remove TR completely from the niosome solution, and there is no detectable signal related to TR in the corresponding chromatogram. The GC-MS results are reported in Fig. 6d. In spite of several advantages of Bio-Beads, they should be removed after application from the vesicle suspension. Lipid loss is another drawback of Bio-Beads usage. It has been shown that adding the beads in several aliquots could reduce the rate of lipid absorption by Bio-Beads, which will reduce lipid loss. ${ }^{58}$ Thus, to obtain efficient reconstitution and reduce the lipid loss, the amount of Bio-Beads should be adjusted exactly. ${ }^{53}$

\section{Conclusions}

Although niosomes are more economically and chemically stable alternatives to liposomes, there are several membrane features that are unknown for niosomes. Based on our results, the following outcomes are proposed: (1) cholesterol incorporation in niosomes increases the rigidity of the membrane and decreases the permeability, leading to the increased bilayer stability. (2) Cholesterol could change the surface tension and gel-to-liquid crystalline phase transition temperature. (3) Cholesterol incorporation can decrease the cytotoxic and hemolytic effects of niosomes. (4) The niosomes with or without cholesterol could be solubilized completely with TR. However, cholesterol changes the solubilizing parameters and increases the amount of TR required for solubilization. (5) TR could be removed after solubilization from niosomes, and the vesicles could re-form successfully. These findings introduce a new niosome bilayer application for protein reconstitution, vaccine design, smart targeting, and, niosome bilayer characterization purposes.

\section{Conflicts of interest}

There are no conflicts to declare.

\section{Acknowledgements}

This study was supported by a grant (Grant No. 930251) from Kerman University of Medical Sciences and the authors greatly acknowledge the support for the financial support. 


\section{References}

1 N. B. Mahale, P. D. Thakkar, R. G. Mali, D. R. Walunj and S. R. Chaudhari, Adv. Colloid Interface Sci., 2012, 183, 46-54.

2 E. R. Geertsma, N. A. B. N. Mahmood, G. K. SchuurmanWolters and B. Poolman, Nat. Protoc., 2008, 3, 256-266.

3 A. Helenius and K. Simons, Biochim. Biophys. Acta, Rev. Biomembr., 1975, 415, 29-79.

4 H. Ahyayauch, M. I. Collado, F. M. Goñi and D. Lichtenberg, FEBS Lett., 2009, 583, 2859-2864.

5 J.-L. Rigaud, B. Pitard and D. Levy, Biochim. Biophys. Acta, Bioenerg., 1995, 1231, 223-246.

6 T. Inoue, K. Miyakawa and R. Shimozawa, Chem. Phys. Lipids, 1986, 42, 261-270.

7 A. M. Seddon, P. Curnow and P. J. Booth, Biochim. Biophys. Acta, Biomembr., 2004, 1666, 105-117.

8 P. K. Vinson, Y. Talmon and A. Walter, Biophys. J., 1989, 56, 669-681.

9 H. Heerklotz, A. D. Tsamaloukas and S. Keller, Nat. Protoc., 2009, 4, 686-697.

10 J. s. Sot, M. I. Collado, J. L. R. Arrondo, A. Alonso and F. 1. M. Gogoi, Langmuir, 2002, 18, 2828-2835.

11 A. V. Popova and D. K. Hincha, Biophys. J., 2007, 93, 12041214.

12 E. Schnitzer, D. Lichtenberg and M. M. Kozlov, Chem. Phys. Lipids, 2003, 126, 55-76.

13 I. Kyrikou, A. Georgopoulos, S. Hatziantoniou, T. Mavromoustakos and C. Demetzos, Chem. Phys. Lipids, 2005, 133, 125-134.

14 E. Schnitzer, M. M. Kozlov and D. Lichtenberg, Chem. Phys. Lipids, 2005, 135, 69-82.

15 C. Toro, S. A. Sanchez, A. Zanocco, E. Lemp, E. Gratton and G. Gunther, Chem. Phys. Lipids, 2009, 157, 104-112.

16 B. Nasseri, Int. J. Pharm., 2005, 300, 95-101.

17 X.-M. Li, M. M. Momsen, J. M. Smaby, H. L. Brockman and R. E. Brown, Biochemistry, 2001, 40, 5954-5963.

18 C. Kirby, J. Clarke and G. Gregoriadis, Biochem. J., 1980, 186, 591-598.

19 A. Genz, J. F. Holzwarth and T. Y. Tsong, Biophys. J., 1986, 50, 1043-1051.

20 A. Akbarzadeh, R. Rezaei-Sadabady, S. Davaran, S. W. Joo, N. Zarghami, Y. Hanifehpour, M. Samiei, M. Kouhi and K. Nejati-Koshki, Nanoscale Res. Lett., 2013, 8, 102.

21 I. F. Uchegbu and S. P. Vyas, Int. J. Pharm., 1998, 172, 33-70.

22 R. M. Handjani-Vila, A. Ribier, B. Rondot and G. Vanlerberghie, Int. J. Cosmet. Sci., 1979, 1, 303-314.

23 R. Rajera, K. Nagpal, S. K. Singh and D. N. Mishra, Biol. Pharm. Bull., 2011, 34, 945-953.

24 M. C. A. Stuart and E. J. Boekema, Biochim. Biophys. Acta, Biomembr., 2007, 1768, 2681-2689.

25 A. Hildebrand, K. Beyer, R. Neubert, P. Garidel and A. Blume, J. Colloid Interface Sci., 2004, 279, 559-571.

26 D. Lichtenberg, H. Ahyayauch, A. Alonso and F. Goñi, Trends Biochem. Sci., 2013, 38, 85-93.

27 A. J. Baillie, A. T. Florence, L. R. Hume, G. T. Muirhead and A. Rogerson, J. Pharm. Pharmacol., 1985, 37, 863-868.
28 J. Varshosaz, A. Pardakhty, V.-i. Hajhashemi and A. R. Najafabadi, Drug Delivery, 2003, 10, 251-262.

29 H. Strahl, F. Burmann and L. W. Hamoen, Nat. Commun., 2014, 5, 3442.

30 O. A. Kucherak, S. Oncul, Z. Darwich, D. A. Yushchenko, Y. Arntz, P. Didier, Y. M. Mély and A. S. Klymchenko, J. Am. Chem. Soc., 2010, 132, 4907-4916.

31 R. N. McElhaney, Chem. Phys. Lipids, 1982, 30, 229-259.

32 D. A. Kendall and R. C. MacDonald, J. Biol. Chem., 1982, 257, 13892-13895.

33 M. Mehrabani, M. Najafi, T. Kamarul, K. Mansouri, M. Iranpour, M. H. Nematollahi, M. Ghazi-Khansari and A. M. Sharifi, Cell Proliferation, 2015, 48, 532-549.

34 B. C. Evans, C. E. Nelson, S. Y. Shann, K. R. Beavers, A. J. Kim, H. Li, H. M. Nelson, T. D. Giorgio and C. L. Duvall, J. Visualized Exp., 2013, e50166.

35 F. M. Goñi and A. Alonso, Biochim. Biophys. Acta, Biomembr., 2000, 1508, 51-68.

36 D. Lichtenberg, R. J. Robson and E. A. Dennis, Biochim. Biophys. Acta, Rev. Biomembr., 1983, 737, 285-304.

37 Y. Roth, E. Opatowski, D. Lichtenberg and M. M. Kozlov, Langmuir, 2000, 16, 2052-2061.

38 D. Lichtenberg, E. Opatowski and M. M. Kozlov, Biochim. Biophys. Acta, Biomembr., 2000, 1508, 1-19.

39 M. C. A. Stuart, J. C. van de Pas and J. B. F. N. Engberts, J. Phys. Org. Chem., 2005, 18, 929-934.

40 S. Mukherjee, H. Raghuraman and A. Chattopadhyay, Biochim. Biophys. Acta, Biomembr., 2007, 1768, 59-66.

41 M. Poss, T. Couch, A. Odufu, J. McCann, J. Mellon, B. Melnick and D. Jenke, J. Chromatogr. Sci., 2003, 41, 410417.

42 H. Abdelkader, S. Ismail, A. Kamal and R. G. Alany, J. Pharm. Sci., 2010, 65, 811-817.

43 T. M. Allen and L. G. Cleland, Biochim. Biophys. Acta, Biomembr., 1980, 597, 418-426.

44 A. F. Azarbayjani, A. Jouyban and S. Y. Chan, J. Pharm. Pharm. Sci., 2009, 12, 218-228.

45 E. I. Vargha-Butler, M. Foldvari and M. Mezei, Colloids Surf., 1989, 42, 375-389.

46 K. J. Fritzsching, J. Kim and G. P. Holland, Biochim. Biophys. Acta, Biomembr., 2013, 1828, 1889-1898.

47 P. J. Davis and K. M. W. Keough, Biochemistry, 1983, 22, 6334-6340.

48 M. Hömberg and M. Müller, J. Chem. Phys., 2010, 132, $04 \mathrm{~B} 609$.

49 S. G. Clerc and T. E. Thompson, Biophys. J., 1995, 68, 23332341.

50 M. Tabbakhian, S. Daneshamouz, N. Tavakoli and M. R. Jaafari, Iran. J. Pharm. Sci., 2005, 1, 119-130.

51 H. E. J. Hofland, J. A. Bouwstra, J. C. Verhoef, G. Buckton, B. Z. Chowdry, M. Ponec and H. E. Junginger, J. Pharm. Pharmacol., 1992, 44, 287-294.

52 D. Noudeh, P. Khazaeli, S. Mirzaei, F. Sharififar and S. Nasrollahosaiani, J. Biol. Sci., 2009, 9, 423.

53 D. Levy, A. Bluzat, M. Seigneuret and J.-L. Rigaud, Biochim. Biophys. Acta, Biomembr., 1990, 1025, 179-190. 
54 O. Lambert, D. Levy, J.-L. Ranck, G. Leblanc and J.-L. Rigaud, Biophys. J., 1998, 74, 918-930.

55 D. D. Lasic, Biochem. J., 1988, 256, 1.

56 D. Lichtenberg, H. Ahyayauch and F. M. Goñi, Biophys. J., 2013, 105, 289-299.
57 J. L. Rigaud, D. Levy, G. Mosser and O. Lambert, Eur. Biophys. J., 1998, 27, 305-319.

58 E. Buxbaum, Biophysical chemistry of proteins: an introduction to laboratory methods, Springer Science \& Business Media, 2010. 